\title{
A Phase II Trial of Osimertinib as the First-Line Treatment of Non-Small Cell Lung Cancer Harboring Activating EGFR Mutations in Circulating Tumor DNA: LiquidLung-0-Cohort 1
}

\author{
Cheol-Kyu Park ${ }^{1}$, Hyun-Ju Cho ${ }^{1}$, Yoo-Duk Choi ${ }^{2}$, In-Jae Oh', Young-Chul Kim \\ ${ }^{1}$ Department of Internal Medicine, Chonnam National University Hwasun Hospital, Hwasun, ${ }^{2}$ Department of Pathology, Chonnam National University \\ Medical School, Gwangju, Korea
}

\begin{abstract}
Purpose Osimertinib is a potent, irreversible third-generation epidermal growth factor receptor (EGFR) tyrosine kinase inhibitor for both EGFR-activating and T790M resistant mutation. The treatment efficacy of osimertinib was assessed in previously untreated patients with metastatic non-small cell lung carcinoma (NSCLC) harboring activating EGFR mutations in circulating tumor DNA (ctDNA) as well as tumor DNA.
\end{abstract}

Materials and Methods Patients with activating EGFR mutations in their tumor DNA underwent screening with ctDNA analysis using Mutyper and Cobas v2 assays. Enrolled subjects received osimertinib $80 \mathrm{mg}$, once daily. Primary endpoint was objective response rate (ORR) and secondary endpoints were ctDNA test sensitivity, progression-free survival (PFS), duration of response (DoR), and safety.

Results Among 39 screened patients, 29 were ctDNA positive for activating EGFR mutations and 19 were enrolled (ex19del, $\mathrm{n}=11$; L858R/L861Q, n=7; G719A, n=1). Median age was 70 and most patients had brain metastases (15/19, 79\%). ctDNA test sensitivity for activating EGFR mutations was 74\% using both methods and 62\% (Mutyper) or 64\% (Cobas v2) for individual methods. ORR was $68 \%$ (13/19), median PFS was 11.1 months (95\% confidence interval [Cl], 0.0 to 26.7$)$, and median DoR was 17.6 months (95\% Cl, 3.5 to 31.7). ORR and median PFS were significantly superior with ex19del (91\%; 21.9 months; $95 \% \mathrm{Cl}, 5.5$ to 38.3$)$ than with L858R/ L861Q (43\%; 5.1 months; $95 \%$ Cl, 2.3 to 7.9). One patient discontinued the drug because of drug-related interstitial pneumonitis.

Conclusion Osimertinib had favorable efficacy in the first-line treatment of metastatic NSCLC harboring activating EGFR mutations in ctDNA as well as tumor DNA.

Key words Osimertinib, EGFR mutation, Circulating tumor DNA, Non-small cell lung carcinoma

\section{Introduction}

Osimertinib is a potent, irreversible third-generation epidermal growth factor receptor tyrosine kinase inhibitor (EGFR-TKI) for both EGFR-activating and T790M resistant mutations [1-7]. In the FLAURA and AURA trials, osimertinib showed promising efficacy in treatment-naïve non-small cell lung cancer (NSCLC) patients with activating EGFR mutations and had a favorable safety profile compared with the first-generation EGFR-TKIs [5-8]. In tissue-based assays, the objective response rate (ORR) ranged from $67 \%$ to $80 \%$, the median duration of response (DoR) ranged from 17.2 to 19.3 months, and the median progression-free survival (PFS) ranged from 18.9 to 22.1 months in first-line treatment with osimertinib $(80 \mathrm{mg})$. Furthermore, previous studies showed improved activity and efficacy of osimertinib against brain metastasis, compared with platinum-based chemotherapy or standard EGFR-TKIs [4,5,8-10]. Thus, osimertinib is currently becoming the standard of care for the first-line treatment of EGFR-mutated NSCLC.

Circulating tumor DNA (ctDNA) can be used as a suitable substitute for tumor DNA in the analysis of EGFR-activating and T790M mutation status. Moreover, plasma genotyping using ctDNA has been shown to be a highly sensitive and specific technique for the detection of EGFR mutations, with an excellent positive predictive value [11-15]. In our previous study, in which osimertinib was administered as a second-line treatment, the sensitivity of plasma ctDNA tests for EGFRactivating and T790M mutations was 65\% (52/80) and 57\% $(21 / 37)$, respectively, and osimertinib showed favorable ORR $(67 \%)$ [14], with almost equivalent efficacy to that of tissue analysis. In a recent report on tissue and plasma EGFR muta-

Correspondence: Young-Chul Kim

Department of Internal Medicine, Chonnam National University Hwasun Hospital, 322 Seoyang-ro, Hwasun 58128, Korea

Tel: 82-61-379-7614 Fax: 82-61-379-7619 E-mail: kyc0923@jnu.ac.kr

Received May 14, 2020 Accepted September 17, 2020 Published Online September 21, 2020

*This data was presented at the World Conference on Lung Cancer, Toronto, 2018. 
tion analysis in the FLAURA trial, the sensitivity of plasma tests for the detection of exon 19 deletion (ex19del) and the L858R mutation was $79 \%$ and $68 \%$, respectively [16].

Although osimertinib showed favorable efficacy in NSCLC patients with EGFR-activating and T790M resistant mutations detected in ctDNA, there is a concern that patients with tumor DNA shedding tend to have a poorer outcome compared to those without tumor DNA shedding. In subgroup analysis of FLAURA and AURA3 trials, the median PFS in the osimertinib arm was shorter in ctDNA-positive vs. -negative patients (FLAURA, 15.2 months vs. 23.5 months; AURA3 trials, 8.2 months vs. 10.1 months) $[4,16]$. In an exploratory analysis in the BELIEF trial of erlotinib and bevacizumab, detection of EGFR mutations in ctDNA was associated with poor survival at any time point during treatment (baseline, response evaluation, and progression) [17].

This trial aimed to assess the treatment efficacy of osimertinib in previously untreated patients with these metastatic NSCLCs which shed the tumor EGFR-activating mutations into circulation.

\section{Materials and Methods}

\section{Trial design and subjects}

This was a phase II, open-label, single-center study of osimertinib in EGFR-TKI-naïve patients with metastatic NSCLC harboring activating EGFR mutations detected in both ctDNA and tumor DNA. Between February 2017 and March 2018, we recruited patients who were histologically or cytologically diagnosed with NSCLC harboring activating EGFR mutations (ex19del, L858R, L861Q, and G719X), but without prior exposure to EGFR-TKIs.

All patients were required to provide a blood sample at screening, to test for activating EGFR mutations in plasma. Sample collection and subsequent ctDNA analysis were performed as described for the ctDNA T790M mutation cohort (Liquid-Lung-O Cohort 2) [14]. Fifteen milliliters of peripheral blood was withdrawn and centrifuged immediately before storage. ctDNA extraction and EGFR mutation analysis were performed using the PANA Mutyper R EGFR assay (Mutyper, Panagene Inc., Daejeon, Korea), based on peptide nucleic acid (PNA)-mediated polymerase chain reaction (PCR) clamping and melting technology and the Cobas EGFR Mutation Test v2 (Cobas v2, Roche Molecular Systems, Pleasanton, CA), based on a real-time PCR technique. Patients who harbored an activating EGFR mutation, detected by either of the two methods, were enrolled in this trial. For genotyping tumor tissue and cytology samples, the PNA Clamp EGFR mutation detection kit (Panagene Inc.) was used (Fig. 1).

Eligibility criteria are provided in detail in Supplementa- ry Material, and key criteria are as follows: age $>18$ years, locally advanced or metastatic NSCLC, no prior exposure to EGFR-TKIs, activating EGFR mutations detected from tumor tissue or cytology specimen and ctDNA by Mutyper or Cobas test, World Health Organization performance status of $0-2$, and a life expectancy $\geq 12$ weeks. Patients with central nervous system (CNS) metastases were enrolled if their disease was asymptomatic or stable after local therapy, including surgery or radiotherapy, before the first dose of osimertinib.

The trial was registered at clinicaltrials.gov (NCT02769286).

\section{Trial procedure, assessment, and treatment}

Eligible patients received $80 \mathrm{mg}$ of osimertinib once a day, regardless of food ingestion, and treatment continued until disease progression, as defined by the Response Evaluation Criteria in Solid Tumors (RECIST) ver. 1.1 [18]; unacceptable toxicity; or another valid reason for ceasing treatment occurred. Response evaluation was performed every 8 weeks for the first three assessments ( 24 weeks), followed by every 12 weeks for subsequent assessments. Regular brain imaging was performed in patients with known brain metastases. For the rest of the patients, brain imaging was only performed when there were symptoms or signs of suspected CNS metastasis. Patients could continue osimertinib after RECIST 1.1-defined progression if they maintained clinical benefits, as assessed by an investigator.

The primary objective was determining the ORR (assessed by RECIST 1.1) of osimertinib in EGFR-TKI-naïve NSCLC patients with activating EGFR mutations detected in ctDNA. The secondary objective was to compare the sensitivity of the Mutyper and Cobas v2 methods for detecting EGFR mutations in ctDNA. In addition, PFS, DoR, and the safety profile with tolerability parameters of osimertinib were measured. PFS was defined as the time (in months) from the first dose of osimertinib until objective disease progression or death, regardless of whether the patient was withdrawn from therapy or received another anticancer therapy prior to progression. DoR was defined as the time (in months) from the documentation of the tumor response to disease progression or death in a patient who had the best overall complete or partial remission response.

Adverse events (AEs) were measured from the beginning of drug administration, throughout the treatment period, until 28 days after the last dose of osimertinib. AEs were graded according to the National Cancer Institute Common Terminology Criteria for Adverse Events (CTCAE), ver. 4.0. If a patient experienced an $\mathrm{AE}$ of CTCAE grade 3 or higher and/or unacceptable toxicity (any grade) that was considered to be associated with osimertinib, dosing could be interrupted for up to 3 weeks. If the toxicity resolved or reverted 


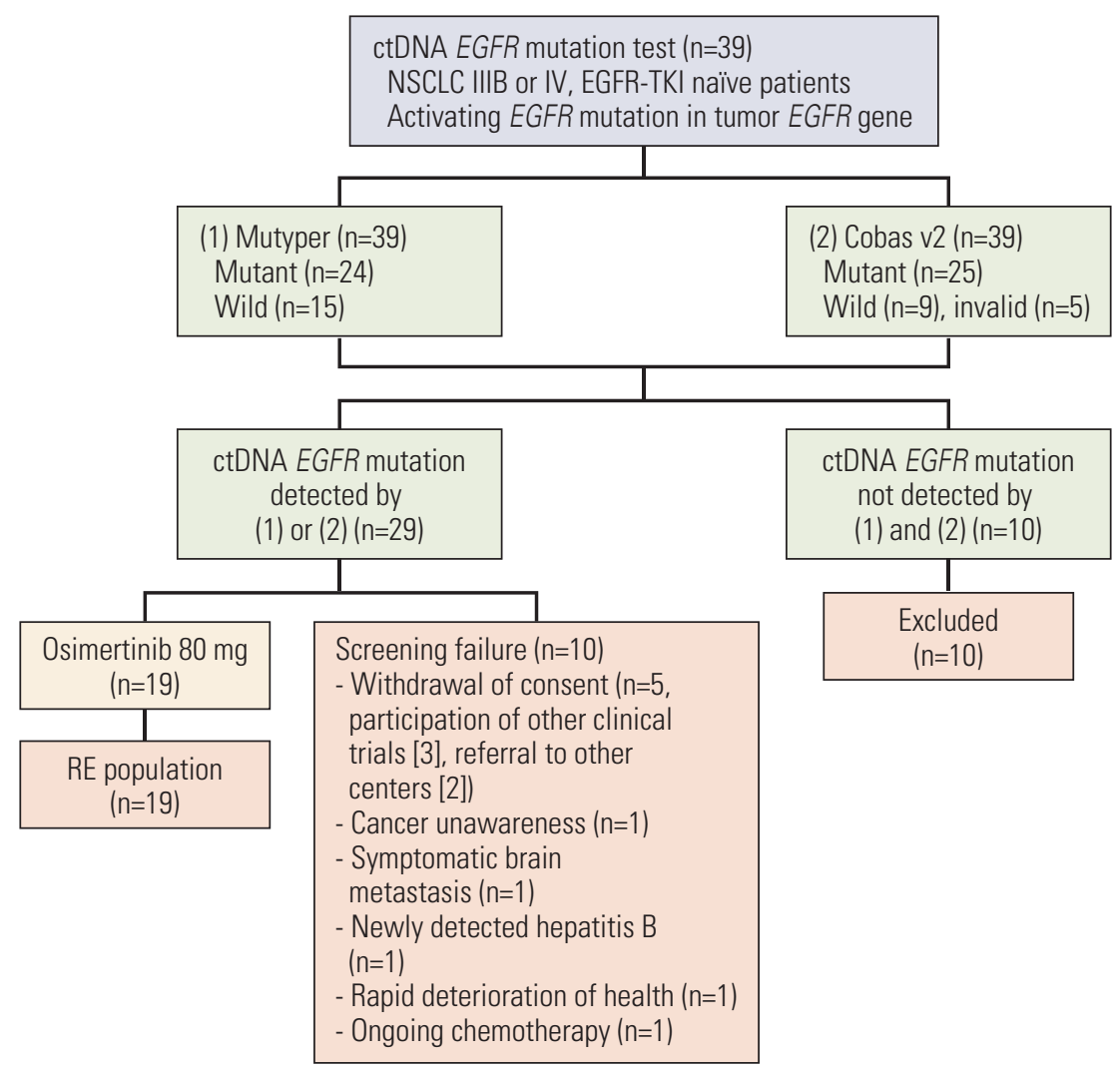

Fig. 1. Study subjects. ctDNA, circulating tumor DNA; EGFR-TKIs, epidermal growth factor receptor tyrosine kinase inhibitors; NSCLC, non-small cell lung cancer; RE, response-evaluable.

to CTCAE grade $\leq 2$ within 3 weeks of onset, osimertinib could be restarted at the same dose ( $80 \mathrm{mg}$, daily) or a lower dose (40 mg, daily), excluding cases with any grade of pulmonary toxicity, symptomatic corrected QT interval prolongation, or corneal ulceration. Once a dose had been reduced, it was not increased in future cycles.

\section{Statistical analysis}

To prove a $60 \%$ ORR of osimertinib, compared to the $30 \%$ ORR in the null hypothesis, and considering a 10\% drop-out rate, 19 patients were required to be enrolled in this study (H0 proportion, 0.3; $\mathrm{H} 1$ proportion, 0.6; sample size, 17; power, 0.8; significance, 0.05; one-sided). The value of the ORR in the null hypothesis (30\%) was based on the ORR of platinum doublet chemotherapy as first-line treatment in cases where the EGFR mutation was not detected by tumor or ctDNA genotyping. The sensitivity of the EGFR mutation test using ctDNA was expected to be $60 \%$ among the screening population [19-21]. Thus, it was calculated that 32 patients $(19 / 0.6=32)$ needed to be screened.

Baseline characteristics and safety data were analyzed in an intent-to-treat (ITT) population, which was defined as the subjects who had received at least one dose of treatment and for whom AEs were monitored ( $n=19)$ (Fig. 1). According to the study protocol, the ORR was assessed in the responseevaluable (RE) population, defined as subjects who had received at least one dose of treatment and for whom response evaluations were available. PFS and DoR were analyzed in the ITT population. In this study, the ITT and RE populations were identical.

Intergroup comparisons were performed using a MannWhitney U test for continuous variables and Pearson's chisquare or Fisher exact tests for categorical variables. Survival times were estimated for each group using the Kaplan-Meier method. Statistical analyses were performed using SPSS ver. 25 (IBM Corp., Armonk, NY) and p-values < 0.05 were used to indicate statistical significance.

\section{Results}

\section{Patient characteristics}

Thirty-nine patients with EGFR-activating mutations detected in tumor DNA were screened from February 2017 
Table 1. Baseline characteristics

\begin{tabular}{|c|c|}
\hline Characteristic & Value $(n=19)$ \\
\hline Age (yr) & $70(32-84)$ \\
\hline Sex (female/male) & $13(68) / 6(32)$ \\
\hline Never smokers & $16(84)$ \\
\hline \multicolumn{2}{|l|}{ WHO performance status ${ }^{a)}$} \\
\hline $0 / 1 / 2$ & $1(5) / 16(84) / 2(11)$ \\
\hline Histology, adenocarcinoma & $19(100)$ \\
\hline Stage IV (by 8th TNM) & $19(100)$ \\
\hline Within the thoracic cavity (M1a) & $1(5)$ \\
\hline Single extrathoracic metastasis ${ }^{\mathrm{b})}(\mathrm{M} 1 \mathrm{~b})$ & $3(16)$ \\
\hline Multiple extrathoracic metastases (M1c) & $15(79)$ \\
\hline Brain metastasis & $15(79)$ \\
\hline Leptomeningeal metastasis & $1(5)$ \\
\hline Brain RT before start of osimertinib & $7(37)$ \\
\hline No preceding brain RT (reasons) & $8(42)$ \\
\hline Small lesion & 6 \\
\hline Old age with poor performance & 2 \\
\hline Asymptomatic & 2 \\
\hline Line of treatment, 1st & $19(100)$ \\
\hline \multicolumn{2}{|l|}{ Type of EGFR mutation ${ }^{\mathrm{c}}$} \\
\hline ex19del & $11(58)$ \\
\hline L858R/L861Q & $7(37)$ \\
\hline G719A & $1(5)$ \\
\hline
\end{tabular}

Values are presented as median (range) or number (\%). EGFR, epidermal growth factor receptor; RT, radiotherapy. ${ }^{\text {a) }} \mathrm{A}$ World Health Organization (WHO) performance status of 0 indicates that the patient is fully active and able to carry out all normal activities without restrictions. A WHO performance status of 1 indicates that the patient is restricted in physically strenuous activity, but is ambulatory and able to carry out work of a light or sedentary nature, such as light housework or office work. A WHO performance status of 2 indicates that the patient is ambulatory and capable of all self-care, but unable to carry out any work activities for more than $50 \%$ of waking hours, ${ }^{\text {b) }}$ All cases were brain metastases, ${ }^{c}$ Results of the tissue-based test at diagnosis. to March 2018. The ctDNA of 29 patients was found to be positive for an EGFR-activating mutation and 19 patients were finally enrolled (ex19del, $n=11 ; L 858 R / L 861 Q, n=7$; G719A, $n=1$ ). Ten patients were excluded for the following reasons: withdrawal of consent $(n=5)$, cancer unawareness $(n=1)$, symptomatic brain metastasis $(n=1)$, newly detected hepatitis $B(n=1)$, rapid deterioration of health $(n=1)$, and ongoing chemotherapy ( $n=1)$ (Fig. 1). Among them, six patients received other EGFR-TKI therapy (gefitinib in five patients and afatinib in a patient).

Baseline characteristics of the enrolled patients are shown in Table 1. The median age was 70 years (range, 32 to 84 years) and female and patients without a history of smoking were dominant. The majority of patients had brain metastases $(15 / 19,79 \%)$ and approximately half of these patients $(7 / 15)$ received brain radiotherapy before the initiation of osimertinib.

\section{Sensitivity of tests for detecting activating EGFR muta- tions in ctDNA}

Activating EGFR mutations were detected using both Mutyper and Cobas v2 methods in 20 cases, only using the Mutyper method in four cases, and only using the Cobas v2 method in five cases (Table 2). There were two cases with discordant mutation types in ctDNA compared with the tissue genotyping results (case 1 with the Mutyper method and case 2 with both methods). The sensitivity of the ctDNA test for activating EGFR mutations was $74 \%$ when using both tests, $62 \%$ when using only the Mutyper test, and $64 \%$ when using only the Cobas v2 test. The concordance rate between two tests in 19 enrolled patients was 68\% (13/19) (Table 3). Discordant cases were as follows: wild-type or invalid result in one test $(n=4)$, different mutation type $(n=2)$ which were discordant with the result of tissue genotyping.

\section{Response and survival}

The response to osimertinib was evaluable in all enrolled

Table 2. Sensitivity of activating EGFR mutation tests using circulating tumor DNA

\begin{tabular}{|c|c|c|c|c|c|c|}
\hline \multirow{3}{*}{ EGFR mutation } & \multirow{3}{*}{ Tissue } & \multicolumn{5}{|c|}{ Plasma ctDNA } \\
\hline & & \multicolumn{2}{|c|}{ Mutyper } & \multicolumn{3}{|c|}{ Cobas v2 } \\
\hline & & Mutant & Wild-Type & Mutant & Wild-Type & Invalid \\
\hline Mutant & $39(100)$ & $24(62)$ & $15(39)$ & $25(64)$ & $9(23)$ & $5(13)$ \\
\hline \multicolumn{7}{|l|}{ Discordant with tissue } \\
\hline Case 1 & ex19del & ex19del/T790M & - & ex19del & - & - \\
\hline Case 2 & G719X & G719A & - & ex19del & - & - \\
\hline Sensitivity & & $24 / 39(62)$ & - & $25 / 39(64)$ & - & - \\
\hline Sensitivity (Mutyper- or Cobas v2-positive) & & $29 / 39(74)$ & - & - & - & - \\
\hline
\end{tabular}

Values are presented as number (\%). ctDNA, circulating tumor DNA; EGFR, epidermal growth factor receptor. 
Table 3. Concordance of activating EGFR mutation between two tests using circulating tumor DNA

\begin{tabular}{|c|c|c|c|c|c|c|c|}
\hline \multirow{2}{*}{$\begin{array}{l}\text { Concordance in } \\
\text { enrolled patients }\end{array}$} & \multicolumn{7}{|c|}{ Cobas v2 } \\
\hline & ex19del & $\begin{array}{l}\text { L858R/ } \\
\text { L861Q }\end{array}$ & $\begin{array}{c}\text { ex19del/ } \\
\text { T790M }\end{array}$ & G719A & Wild-Type & Invalid & Total \\
\hline \multicolumn{8}{|l|}{ Mutyper } \\
\hline ex19del & 9 & - & - & - & - & - & 9 \\
\hline L858R/L861Q & - & 4 & - & - & 1 & 2 & 7 \\
\hline ex19del/T790M & 1 & - & - & - & - & - & 1 \\
\hline G719A & 1 & - & - & - & - & - & 1 \\
\hline Wild-type & 1 & - & - & - & - & - & 1 \\
\hline Invalid & - & - & - & - & - & - & 0 \\
\hline Total & 12 & 4 & 0 & 0 & 1 & 2 & 19 \\
\hline
\end{tabular}

EGFR, epidermal growth factor receptor.

patients. Partial remissions were observed in 13 cases (ORR $68 \%$ ) and the disease control rate (DCR) was 95\% (Table 4, Fig. 2). According to EGFR mutation subtype identified by tumor genotyping, patients with the ex19del mutation showed more favorable ORR than those with L858R/L861Q mutations (91\% vs. $43 \%, \mathrm{p}=0.032)$. In patients with known brain metastasis, the DCR of the brain metastasis was $100 \%$ (15/15).

The final analysis of PFS and DoR was performed at 79\% maturity (15/19). The data cut-off date was December 4, 2019 and the median follow-up duration was 25.0 months (95\% confidence interval [CI], 23.9 to 26.1). The median PFS and DoR of the enrolled patients were 11.1 months (95\% CI, 0.0 to 26.7 ) and 17.6 months (95\% CI, 3.5 to 31.7$)$. Excluding the patients with concomitant EGFR mutation and anaplastic lymphoma kinase $(A L K)$ rearrangement $(n=2)$, ORR was $77 \%$ (13/17) and median PFS was 19.2 months (95\% CI, 5.9 to 32.5). Most patients showed objective responses within two cycles of osimertinib. The ORR was greater and PFS time was longer in patients without brain metastasis than in patients with brain metastasis (Table 4, S1A Fig.), but the difference was not statistically significant. Based on tumor genotyping, patients with the ex19del mutation (21.9 months; 95\% CI, 5.5 to 38.3) showed a more favorable median PFS than patients with the L858R/L861Q mutations (5.1 months; 95\% CI, 2.3 to 7.9; $p=0.004$ ) (S1B Fig.). Data on overall survival were immature at the time of this analysis ( $21 \%$ maturity).

The response to osimertinib was not significantly different according to ctDNA genotyping methods. The ORR and DCR, according to positive results of EGFR mutation tests, were as follows: both Mutyper- and Cobas v2-positive, 73\% and $93 \%$, respectively; Mutyper only, $67 \%$ and $100 \%$, respectively; and Cobas v2 only, $0 \%$ and $100 \%$, respectively ( $p>$ 0.05 ) (Table 4). With the exclusion of cases that were discordant in ctDNA mutation type (Table 2), ORR showed a sig- nificant difference according to ctDNA EGFR mutation type (ex19del, 100\% vs. L858R/L861Q, 25\%; p=0.014).

\section{Pattern of progression and subsequent treatment after osimertinib}

Four patients were still receiving osimertinib at the time of data cut-off, and 15 patients discontinued the first-line treatment due to progression (Fig. 3A). Among the patterns of progression, the growth of the primary tumor was the most common, followed by the aggravation of non-target lesions, such as in the bone, pleura, brain, and lymph nodes. All patients with progression to the brain had brain metastases at baseline (Fig. 3B). In total, nine patients (47\%) started a second-line therapy after the discontinuation of osimertin$\mathrm{ib}$, with cytotoxic chemotherapy and EGFR-TKI other than osimertinib being the most common options (Fig. 3A). Five patients $(26 \%)$ entered a third-line therapy and all of these patients are still being treated (Fig. 3C).

\section{Toxicity and safety}

A summary of the toxicity profiles of all enrolled patients who received osimertinib is shown in Table 5. AEs occurred in 18 of 19 patients $(95 \%)$ and grade 3 or 4 events developed in six patients $(32 \%)$. Only one patient experienced drugrelated interstitial pneumonitis of grade 3, and this resulted in drug discontinuation. The majority of AEs were gastrointestinal and skin manifestations. All AEs associated with osimertinib are described in Table 5.

\section{Discussion}

This was a prospective trial for evaluating the treatment efficacy of osimertinib in previously untreated patients with advanced or metastatic NSCLC, harboring activating EGFR 


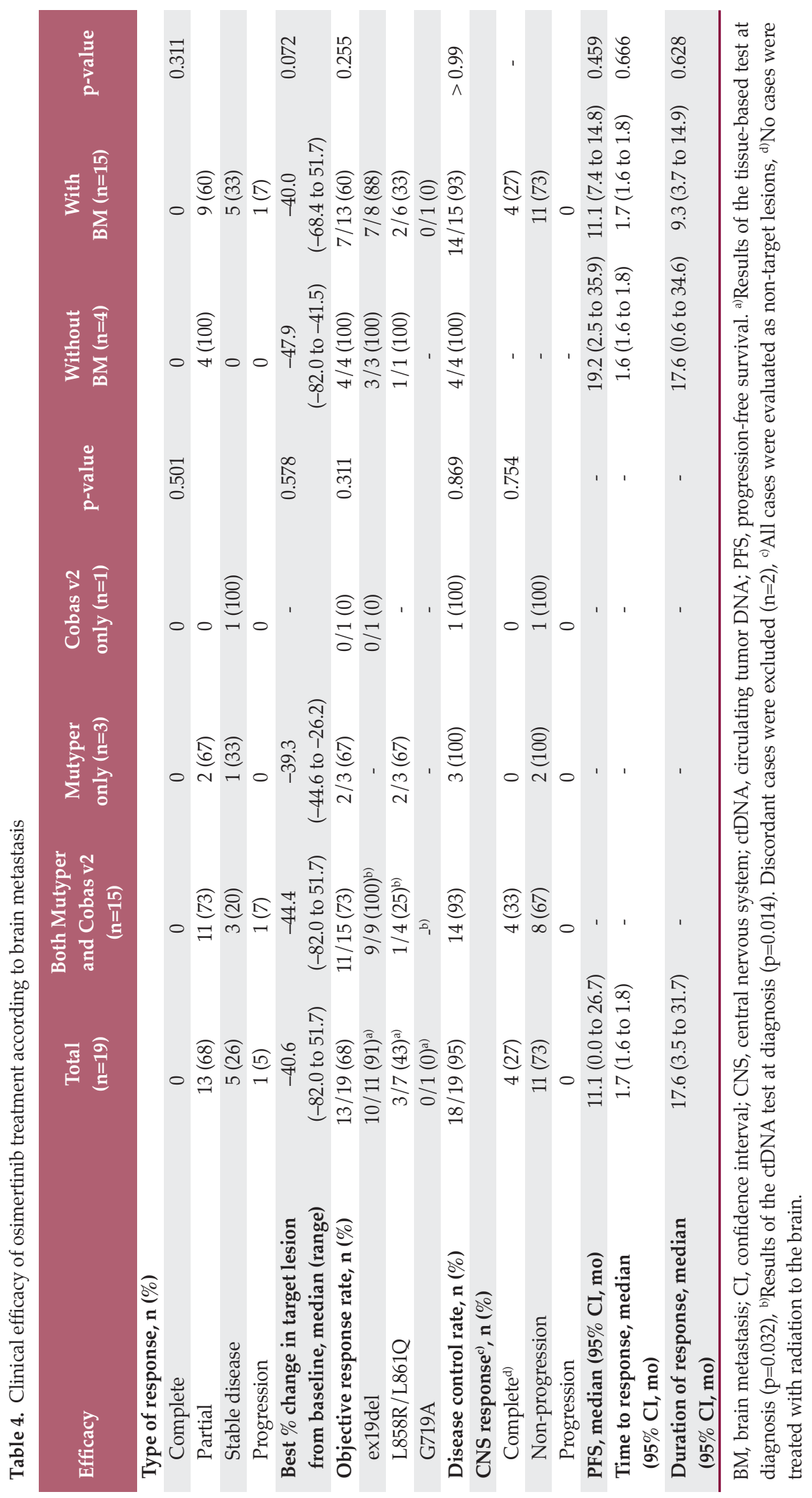




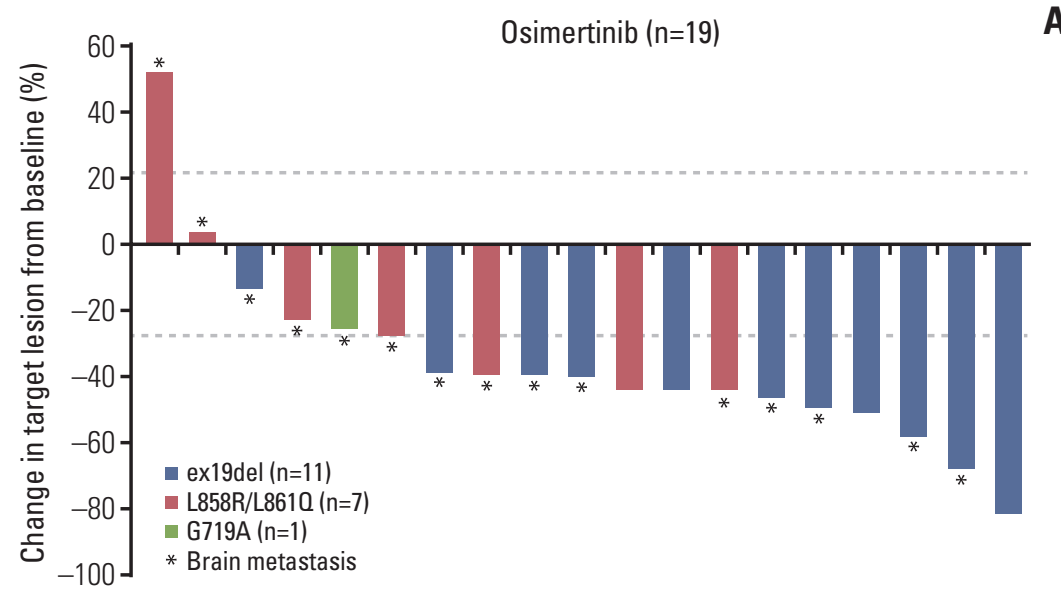

A

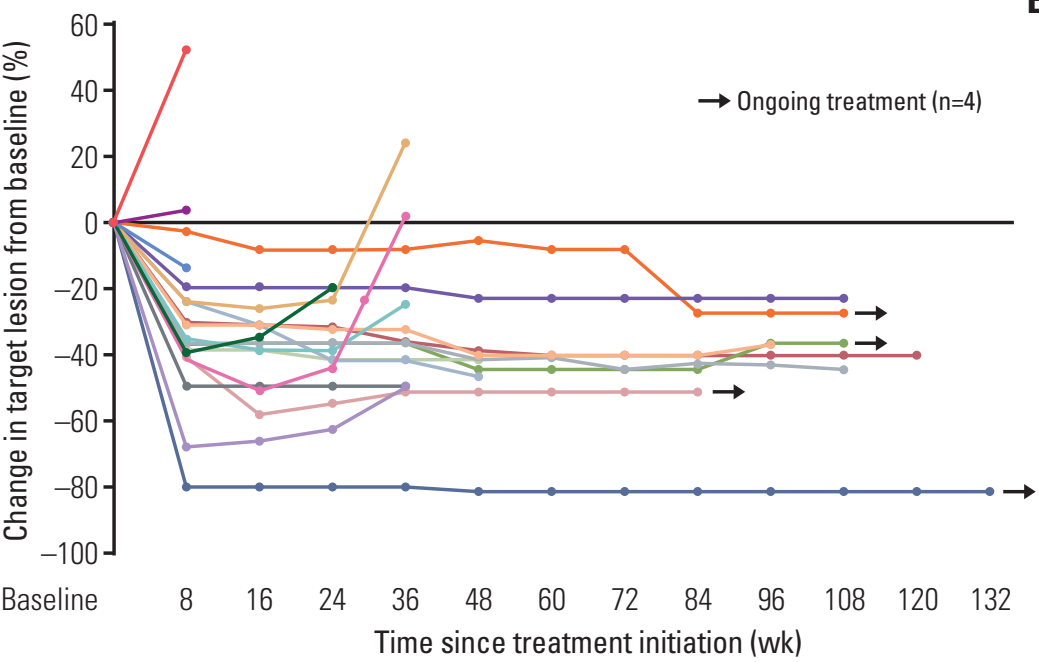

Fig. 2. Response to osimertinib. Waterfall plot (A) and Spider plot (B), according to the Response Evaluation Criteria in Solid Tumors.

mutations in both ctDNA and tumor DNA. In the present study, osimertinib showed favorable efficacy and a safety profile similar to that from previous trials assessing osimertinib as the first-line treatment of EGFR-mutated NSCLC [5-8].

An allele-specific PCR assay (Cobas v2) is the only FDAapproved assay for plasma EGFR genotyping, and recent advances in highly sensitive technologies have led to the development of novel assays using ctDNA [22]. In the present study, we performed PCR-based analysis of activating EGFR mutations. The sensitivity of the ctDNA tests was $74 \%$ when using both methods and 62\% (Mutyper) or $64 \%$ (Cobas v2) when using either method individually. This study showed higher sensitivity for activating EGFR mutations compared with our previous trial (65\%) [14] and a similar result to that by ctDNA analysis in the FLAURA trial (68\%-79\%) [16]. Taken together, we demonstrated that plasma EGFR genotyping can be a screening tool for the de- tection of activating EGFR mutations at initial diagnosis, as well as T790M resistance mutations at progression after prior EGFR-TKI treatment.

There was a case with discordant mutation type in ctDNA between Mutyper (G719A) and Cobas v2 (ex19del), while the result of tissue genotyping was G719X, thus Cobas testing might need a quality control procedure. However, all other cases showed concordant results between the tests and the positive results were translated into clinical efficacy. Considering a previous study of Oxnard et al. [15] highlighting that a well-validated assay has negligible false positives and the clinical outcome would be the best reference standard for a non-invasive assay, the discrepancy may also be explained by a combined EGFR mutation attributed to intratumoural heterogeneity.

In the demographics of the present study, the median age (70 vs. 64) and the proportion of patients with known brain 
A

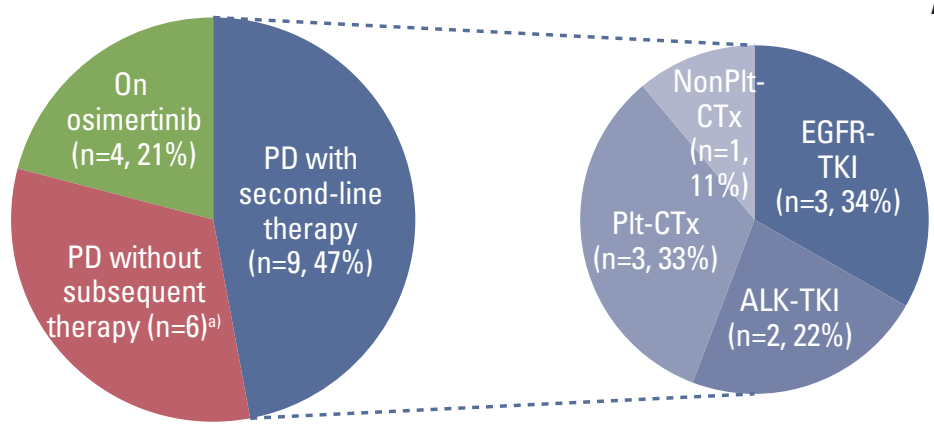

B
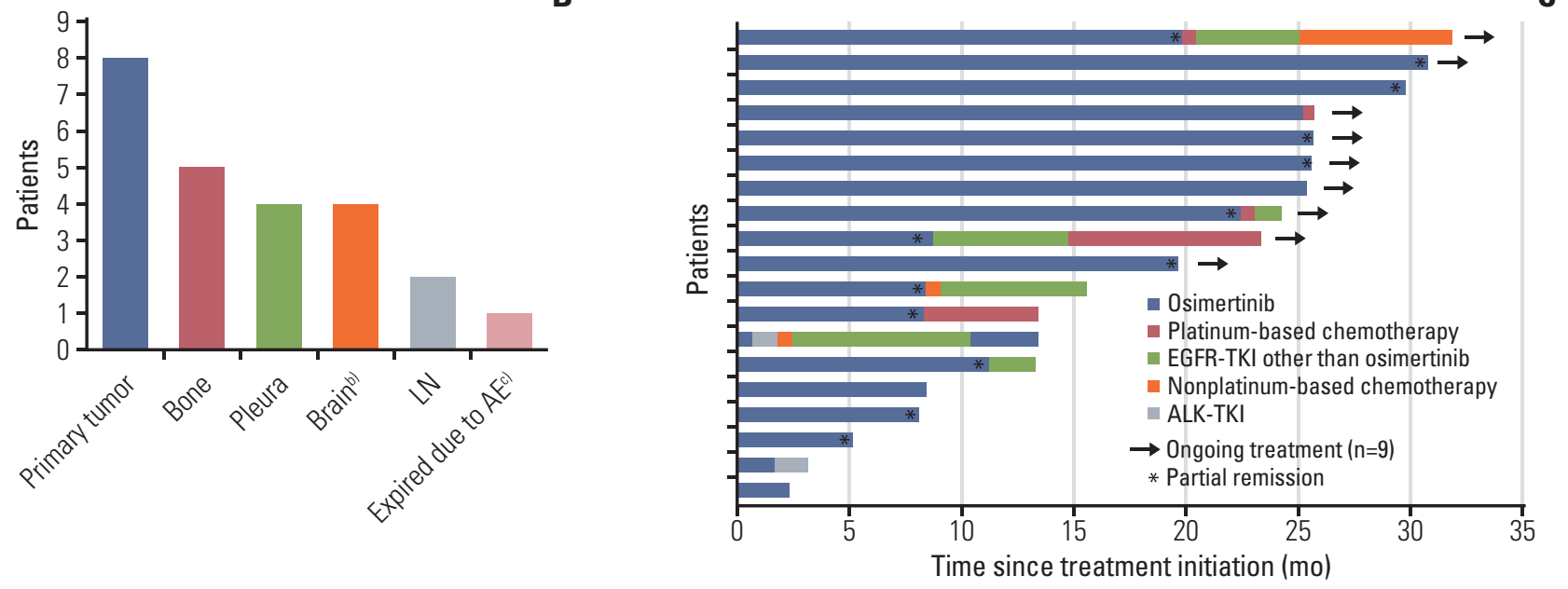

Fig. 3. Subsequent treatment after osimertinib. Overview of the pattern of progression and subsequent treatment after osimertinib. Treatment status of enrolled patients and sequential treatment following first-line osimertinib (A). Patterns of progression after osimertinib (B). The numbers in parentheses refer to the corresponding number of patients. Swimmer plot for sequential treatment after osimertinib (C). $\mathrm{AE}$, adverse event; ALK, anaplastic lymphoma kinase; CTx, chemotherapy; EGFR, epidermal growth factor receptor; LN, lymph node; PD, progressive disease; Plt, platinum; TKI, tyrosine kinase inhibitor. ${ }^{a}$ Best supportive care $(n=4)$, death $(n=1)$, referral to another center $(n=1)$, ${ }^{b}$ All patients had brain metastasis at baseline, ${ }^{c}$ Death due to drug-related interstitial pneumonia.

metastasis (79\% vs. 19\%) were higher compared with that in the osimertinib arm of the FLAURA trial [5]. In FLAURA and AURA3 trials, osimertinib showed a clear benefit in PFS regardless of CNS metastasis, but there were numerical differences: 19.1 months vs. 15.2 months in FLAURA and 10.8 months vs. 8.5 months in AURA3 [4,5], according to the presence of brain metastases. In the present study, median PFS also showed a numerical difference according to brain metastasis (19.2 months vs. 11.1 months, $\mathrm{p}=0.459)$. Patients with EGFR-mutated NSCLC who have brain metastasis tend to have a poorer prognosis compared to those without brain metastasis [23]. However, as reported in the FLAURA trial, osimertinib showed favorable CNS efficacy, in terms of ORR, PFS, and DoR compared with standard (first-generation) EGFR-TKIs, and the benefit was also shown in patients with suspected leptomeningeal metastasis [10]. In the present study, all patients with progression to the brain after osimertinib treatment had brain metastasis at baseline.

In the subgroup analysis of the FLAURA trial, although there was no difference in hazard ratio (HR) according to the detection of ctDNA EGFR mutations [5], the median PFS in the osimertinib arm was numerically shorter in ctDNA EGFR mutation-positive vs. -negative patients (15.2 months vs. 23.5 months) [16]. This tendency of worse outcomes in shedding tumors was also seen in the osimertinib arm of the AURA3 trial (8.2 months vs. 10.1 months) [4]. This process of ctDNA shedding is known to be related to tumor biology, such as mitotic rate, necrosis, and the overall burden of the metastatic tumor [22].

The median PFS in the present study was shorter than that in the FLAURA trial. This could be associated with the baseline characteristics of the patients enrolled in the present 
Table 5. Safety profile and all adverse events associated with osimertinib treatment

\begin{tabular}{|c|c|c|}
\hline All events & $\begin{array}{c}\text { Any grade }{ }^{a} \\
\quad(n=19)\end{array}$ & $\begin{array}{l}\left.\text { Grade } \geq 3^{a}\right) \\
\quad(n=19)\end{array}$ \\
\hline Adverse events (AEs) & $17(90)$ & $4(21)$ \\
\hline Drug-related AEs & $12(63)$ & $1(5)^{\mathrm{a})}$ \\
\hline AEs leading to dose reduction & 0 & \\
\hline AEs leading to drug discontinuation ${ }^{\text {b) }}$ & $1(5)$ & \\
\hline Serious AEs & $7(37)$ & \\
\hline Serious AEs, drug-related ${ }^{\text {b) }}$ & $1(5)$ & \\
\hline Rash or acne & $3(16)$ & 0 \\
\hline Pruritus & $5(26)$ & 0 \\
\hline Dry skin & $2(11)$ & 0 \\
\hline Mucositis & $5(26)$ & 0 \\
\hline Paronychia & $3(16)$ & 0 \\
\hline Stomatitis & $1(5)$ & 0 \\
\hline Conjunctivitis & $3(16)$ & 0 \\
\hline Epigastric discomfort & $2(11)$ & $1(5)$ \\
\hline Nausea & $2(11)$ & 0 \\
\hline Vomiting & $4(21)$ & 0 \\
\hline Diarrhea & $2(11)$ & 0 \\
\hline Constipation & $2(11)$ & 0 \\
\hline Headache & $3(16)$ & 0 \\
\hline Dizziness & $2(11)$ & $1(5)$ \\
\hline Unconsciousness & $1(5.3)$ & $1(5)$ \\
\hline Seizure & $1(5.3)$ & 0 \\
\hline Back pain & $3(16)$ & 0 \\
\hline LFT $^{\mathrm{c}}$ elevation & $2(11)$ & 0 \\
\hline Anemia & $1(5)$ & $1(5)$ \\
\hline Hypocalcemia & $2(11)$ & $1(5)$ \\
\hline Dyspnea & $2(11)$ & 0 \\
\hline Cough & $1(5)$ & 0 \\
\hline Sputum & $1(5)$ & 0 \\
\hline Rhinorrhea & $2(11)$ & 0 \\
\hline Pneumonitis ${ }^{\text {b) }}$ & $3(16)$ & $2(11)$ \\
\hline Pleural effusion & $1(5)$ & 0 \\
\hline Edema & $1(5)$ & 0 \\
\hline Myalgia & $1(5)$ & 0 \\
\hline Urinary tract infection & $1(5)$ & 0 \\
\hline Hair loss & $1(5)$ & 0 \\
\hline
\end{tabular}

Values are presented as number (\%). LFT, liver function test. a)Patients with multiple events in the same category were only counted once in that category. Patients with events in more than one category were counted once in each category, b)Interstitial pneumonitis, ${ }^{c}$ Aspartate aminotransferase or alanine aminotransferase.

study, the majority of whom were older and had developed brain metastases and all of whom were ctDNA-positive. Based on these characteristics, the survival benefit of osimertinib would be underestimated in the present study; how- ever, this study also provided real-world evidence that osimertinib has favorable efficacy and a tolerable safety profile, even in older patients with brain metastases.

Although the existence of significant differences in the efficacy of EGFR-TKIs between patients with ex19del and L858R mutations may be controversial, most studies of firstand second-generation EGFR-TKIs have demonstrated that patients with ex19del mutations have better ORR and survival rates compared to those with L858R or other uncommon mutations [24,25]. In the FLAURA trial, a subgroup analysis of PFS showed little difference in HR according to EGFR mutation subtype ( 0.43 for ex19del and 0.51 for L858R), while the median PFS in the osimertinib arm was numerically longer in patients with ex19del vs. L858R mutations (21.4 months vs. 14.4 months) [5]. Furthermore, the osimertinib arm showed an OS benefit, compared with standard EGFR-TKIs, in patients with the ex19del mutation (HR, 0.68; 95\% CI, 0.51 to 0.90), while there was no difference in OS in patients with the L858R mutation (HR, 1.00; $95 \%$ CI, 0.71 to 1.40) [7]. However, in a report on two treatmentnaïve cohorts of the AURA phase I trial with different doses of osimertinib (80 mg and $160 \mathrm{mg}$ ), there was no difference in median PFS time according to mutation subtype (ex19del, 23.4 months vs. L858R, 22.1 months) [6]. In the present study, patients with the ex19del mutation showed more favorable ORR and PFS than those with the L858R/L861Q mutations, which was consistent with the results of the FLAURA phase III trial. Therefore, osimertinib may be strongly recommend as the first-line treatment for patients with EGFR-mutated NSCLC, particularly for those harboring the ex19del mutation in tumor tissue or ctDNA.

The patterns of subsequent treatment following first-line osimertinib treatment in the present study were similar to those of the FLAURA trial [7] and were as follows: subsequent therapy, $47 \%$ vs. $48 \%$; no subsequent therapy, $32 \%$ vs. $31 \%$; ongoing osimertinib, $21 \%$ vs. $22 \%$ in the present study and the FLAURA trial, respectively (Fig. 3A). Although two patients received the ALK inhibitor, crizotinib, as the secondline treatment, they had only weakly positive florescence results in in situ hybridization analysis of ALK (15\% and 20\%) and the duration of treatment was only 1.5 and 1.1 months.

In the present study, for the majority of patients who received treatment subsequent to osimertinib, the duration of treatment was less than 6 months (Fig. 3C). In the standard EGFR-TKI arm of the FLAURA trial, only $31 \%$ of patients received osimertinib by cross-over, as the first subsequent therapy [7]. In a real-world study on a Korean cohort, data collected as part of the ASTRIS trial showed that rebiopsy was performed in $60 \%(112 / 188)$ of screened patients. The detection rate for T790M resistance mutations was 53\% (48/90) in rebiopsy tissue from these patients with available EGFR 
mutation analysis, whereas the detection rate was only $30 \%$ $(33 / 112)$ in plasma ctDNA from patients intending to undergo rebiopsy [26]. In addition, $32 \%(6 / 19)$ of patients in the present study discontinued osimertinib and did not receive the first subsequent therapy, owing to deterioration of health or death in approximately $80 \%(5 / 6)$ of these patients. These results suggested that the most effective therapy for patients with advanced EGFR-mutated NSCLC should be the initial treatment. As shown in the swimmer plot (Fig. 3C), most patients who had long-term first-line treatment with osimertinib showed favorable responses initially and remained alive at the end of the follow-up period.

Resistance develops in most EGFR-mutated NSCLC patients receiving EGFR-TKIs as the first-line treatment. As the use of osimertinib is expected to increase, the mechanism of resistance to osimertinib needs to be identified. In the cohort of the phase I component of the AURA trial, genomic resistance mutations, analyzed by next-generation sequencing of plasma samples, were identified in nine patients who received osimertinib as the first-line treatment and then showed progression [6]. The resistance mutations detected in post-progression plasma samples included MET, EGFR, and KRAS amplifications and PIK3CA and KRAS mutations, which was similar to previously reported resistance mechanisms of second-line osimertinib treatment. In the present study, we could not perform tissue rebiopsy in any of the patients who progressed after osimertinib treatment, while ctDNA analysis by plasma reverse transcription polymerase chain reaction was performed in four patients who had the ex19del mutation, detected by tissue genotyping at baseline. In the plasma genotyping results, known mutations (ex19del) remained in two patients, but changed to the wildtype sequence in another two patients. A systematic analysis using a larger plasma sample cohort, combined with tissuebased analysis, would be necessary to clarify these results.

There were several limitations to this study. First, the low number of patients were enrolled although the sample size was calculated based on the rational statistics. The comparison with the results from a randomized phase III trial (FLAURA) might overdraw certain issues for the study populations or the efficacy of osimertinib. Second, a serial follow-up of blood samples was not performed during osimertinib treatment, and the monitoring of response or resist- ance using ctDNA was not attempted by the study protocol. According to an exploratory analysis of FLAURA, clearance of plasma EGFR mutation after 3- or 6-weeks of osimertinib and comparator EGFR-TKI therapy were associated with improvement in PFS [27].

In conclusion, osimertinib had favorable efficacy as the first-line treatment of metastatic NSCLC in individuals harboring activating EGFR mutations, detected in ctDNA as well as tumor DNA. The results of this phase II prospective trial support the up-front use of osimertinib, having favorable efficacy and tolerability, even in elderly patients with brain metastases and tumor DNA shedding into the circulation.

\section{Electronic Supplementary Material}

Supplementary materials are available at Cancer Research and Treatment website (https://www.e-crt.org).

\section{Ethical Statement}

This study was performed in accordance with the Declaration of Helsinki and Good Clinical Practice guidelines and was approved by institutional ethics review boards (CNUHH-2016-065) and the Korean Ministry of Food and Drug Safety (30956). All patients were required to provide written informed consent before participating in this study.

\section{Author Contributions \\ Conceived and designed the analysis: Kim YC. Collected the data: Park CK, Cho HJ, Choi YD, Oh IJ, Kim YC. Contributed data or analysis tools: Park CK, Oh IJ, Kim YC. Performed the analysis: Park CK, Oh IJ, Kim YC. Wrote the paper: Park CK, Cho HJ, Choi YD, Oh IJ, Kim YC.}

\section{Conflicts of Interest}

Panagene and Roche molecular diagnostics, Korea supported this study as providing screening kits for ctDNA extraction and EGFR mutation analysis. Those companies had no role in the study design, data collection and analysis, decision to publish, or preparation of the manuscript.

\section{Acknowledgments}

This research was conducted with support from an Investigator Sponsored Study Programme of AstraZeneca (ESR-15-11075).

\section{References}

1. Cross DA, Ashton SE, Ghiorghiu S, Eberlein C, Nebhan CA, Spitzler PJ, et al. AZD9291, an irreversible EGFR TKI, overcomes T790M-mediated resistance to EGFR inhibitors in lung cancer. Cancer Discov. 2014;4:1046-61.
2. Janne PA, Yang JC, Kim DW, Planchard D, Ohe Y, Ramalingam SS, et al. AZD9291 in EGFR inhibitor-resistant non-small-cell lung cancer. N Engl J Med. 2015;372:1689-99.

3. Yang JC, Ahn MJ, Kim DW, Ramalingam SS, Sequist LV, 
$\mathrm{Su}$ WC, et al. Osimertinib in pretreated T790M-positive advanced non-small-cell lung cancer: AURA study phase II extension component. J Clin Oncol. 2017;35:1288-96.

4. Mok TS, Wu YL, Ahn MJ, Garassino MC, Kim HR, Ramalingam SS, et al. Osimertinib or platinum-pemetrexed in EGFR T790M-positive lung cancer. N Engl J Med. 2017;376:629-40.

5. Soria JC, Ohe Y, Vansteenkiste J, Reungwetwattana T, Chewaskulyong B, Lee $\mathrm{KH}$, et al. Osimertinib in untreated EGFRmutated advanced non-small-cell lung cancer. N Engl J Med. 2018;378:113-25.

6. Ramalingam SS, Yang JC, Lee CK, Kurata T, Kim DW, John T, et al. Osimertinib as first-line treatment of EGFR mutationpositive advanced non-small-cell lung cancer. J Clin Oncol. 2018;36:841-9.

7. Ramalingam SS, Vansteenkiste J, Planchard D, Cho BC, Gray JE, Ohe $Y$, et al. Overall survival with osimertinib in untreated, EGFR-mutated advanced NSCLC. N Engl J Med. 2020;382:41-50.

8. Cho BC, Chewaskulyong B, Lee KH, Dechaphunkul A, Sriuranpong V, Imamura F, et al. Osimertinib versus standard of care EGFR TKI as first-line treatment in patients with EGFRm advanced NSCLC: FLAURA Asian Subset. J Thorac Oncol. 2019;14:99-106.

9. Ballard P, Yates JW, Yang Z, Kim DW, Yang JC, Cantarini M, et al. Preclinical comparison of osimertinib with other EGFRTKIs in EGFR-mutant NSCLC brain metastases models, and early evidence of clinical brain metastases activity. Clin Cancer Res. 2016;22:5130-40.

10. Reungwetwattana T, Nakagawa K, Cho BC, Cobo M, Cho EK, Bertolini A, et al. CNS response to osimertinib versus standard epidermal growth factor receptor tyrosine kinase inhibitors in patients with untreated EGFR-mutated advanced nonsmall-cell lung cancer. J Clin Oncol. 2018;36:3290-7.

11. Douillard JY, Ostoros G, Cobo M, Ciuleanu T, Cole R, McWalter G, et al. Gefitinib treatment in EGFR mutated caucasian NSCLC: circulating-free tumor DNA as a surrogate for determination of EGFR status. J Thorac Oncol. 2014;9:1345-53.

12. Qiu M, Wang J, Xu Y, Ding X, Li M, Jiang F, et al. Circulating tumor DNA is effective for the detection of EGFR mutation in non-small cell lung cancer: a meta-analysis. Cancer Epidemiol Biomarkers Prev. 2015;24:206-12.

13. Thress KS, Brant R, Carr TH, Dearden S, Jenkins S, Brown H, et al. EGFR mutation detection in ctDNA from NSCLC patient plasma: a cross-platform comparison of leading technologies to support the clinical development of AZD9291. Lung Cancer. 2015;90:509-15.

14. Park CK, Cho HJ, Choi YD, Oh IJ, Kim YC. A phase II trial of osimertinib in the second-line treatment of non-small cell lung cancer with the EGFR T790M mutation, detected from circulating tumor DNA: LiquidLung-O-Cohort 2. Cancer Res Treat. 2019;51:777-87.

15. Oxnard GR, Thress KS, Alden RS, Lawrance R, Paweletz CP, Cantarini $\mathrm{M}$, et al. Association between plasma genotyping and outcomes of treatment with osimertinib (AZD9291) in advanced non-small-cell lung cancer. J Clin Oncol. 2016;34: 3375-82.

16. Gray JE, Okamoto I, Sriuranpong V, Vansteenkiste J, Imamura F, Lee JS, et al. Tissue and plasma EGFR mutation analysis in the FLAURA trial: osimertinib versus comparator EGFR tyrosine kinase inhibitor as first-line treatment in patients with EGFR-mutated advanced non-small cell lung cancer. Clin Cancer Res. 2019;25:6644-52.

17. Molina-Vila MA, Stahel RA, Dafni U, Jordana-Ariza N, Balada-Bel A, Garzon-Ibanez M, et al. Evolution and clinical impact of EGFR mutations in circulating free DNA in the BELIEF trial. J Thorac Oncol. 2020;15:416-25.

18. Eisenhauer EA, Therasse P, Bogaerts J, Schwartz LH, Sargent D, Ford R, et al. New response evaluation criteria in solid tumours: revised RECIST guideline (version 1.1). Eur J Cancer. 2009;45:228-47.

19. Weber B, Meldgaard P, Hager H, Wu L, Wei W, Tsai J, et al. Detection of EGFR mutations in plasma and biopsies from non-small cell lung cancer patients by allele-specific PCR assays. BMC Cancer. 2014;14:294.

20. Jenkins S, Yang JC, Ramalingam SS, Yu K, Patel S, Weston S, et al. Plasma ctDNA analysis for detection of the EGFR T790M mutation in patients with advanced non-small cell lung cancer. J Thorac Oncol. 2017;12:1061-70.

21. Choi CM, Kim HC, Jung CY, Cho DG, Jeon JH, Lee JE, et al. Report of the Korean Association of Lung Cancer Registry (KALC-R), 2014. Cancer Res Treat. 2019;51:1400-10.

22. Sacher AG, Komatsubara KM, Oxnard GR. Application of plasma genotyping technologies in non-small cell lung cancer: a practical review. J Thorac Oncol. 2017;12:1344-56.

23. Bhatt VR, D'Souza SP, Smith LM, Cushman-Vokoun AM, Noronha V, Verma V, et al. Epidermal growth factor receptor mutational status and brain metastases in non-small-cell lung cancer. J Glob Oncol. 2017;3:208-17.

24. Sheng M, Wang F, Zhao Y, Li S, Wang X, Shou T, et al. Comparison of clinical outcomes of patients with non-small-cell lung cancer harbouring epidermal growth factor receptor exon 19 or exon 21 mutations after tyrosine kinase inhibitors treatment: a meta-analysis. Eur J Clin Pharmacol. 2016;72:1-11.

25. Yang JC, Wu YL, Schuler M, Sebastian M, Popat S, Yamamoto $\mathrm{N}$, et al. Afatinib versus cisplatin-based chemotherapy for EGFR mutation-positive lung adenocarcinoma (LUX-Lung 3 and LUX-Lung 6): analysis of overall survival data from two randomised, phase 3 trials. Lancet Oncol. 2015;16:141-51.

26. Hong MH, Kim HR, Ahn BC, Heo SJ, Kim JH, Cho BC. Realworld analysis of the efficacy of rebiopsy and EGFR mutation test of tissue and plasma samples in drug-resistant non-small cell lung cancer. Yonsei Med J. 2019;60:525-34.

27. Zhou C, Imamura F, Cheng Y, Okamoto I, Cho BC, Lin MC, et al. Early clearance of plasma EGFR mutations as a predictor of response to osimertinib and comparator EGFR-TKIs in the FLAURA trial. J Clin Oncol. 2019;37(15 Suppl):9020. 